\title{
E-cadherin and 5-HT alterations in the heart of rats having undergone atropine-induced toxicity
}

\author{
QUAN-YONG HUANG ${ }^{1}$, XUE-FANG LI $^{2}$ and SHUI-PING LIU ${ }^{3}$ \\ ${ }^{1}$ Department of Pathology, School of Basic Medical Sciences, Dali University; ${ }^{2}$ School of Basic Medical Sciences, \\ Dali University, Dali, Yunnan 671000; ${ }^{3}$ Department of Forensic Pathology, Zhongshan School of Medicine, \\ Sun Yat-Sen University, Guangzhou 510080, P.R. China
}

Received October 10, 2011; Accepted December 15, 2011

DOI: $10.3892 / \mathrm{mmr} .2011 .727$

\begin{abstract}
Atropine-induced heart damage is associated with changes in the expression of various enzymes and proteins. The purpose of this study was to investigate atropine-induced alterations in cardiac E-cadherin and 5-hydroxytryptamine (5-HT) after atropine administration. Male Wistar rats were randomly divided into two groups: a control group and an atropine group. The atropine group intraperitoneally received atropine at a single dose of $15 \mathrm{mg} / \mathrm{kg}$ for 7 days; the controls received the same amount of saline via the same route. On Day 8, the rats were anesthetized, and a thoracotomy was performed in all animals. Immunohistochemical analysis was performed to evaluate protein expression of E-cadherin and 5-HT. Sections were analyzed by digital image analysis. Cardiac protein expression of E-cadherin and 5-HT was altered after atropine-induced toxicity in the rat. The expression levels of E-cadherin and 5-HT were significantly decreased after atropine treatment, supported by IOD analysis, when compared with the control $(\mathrm{P}<0.05)$. The current findings indicate that such changes would be reflected in abnormal cardiac function, and these proteins may be useful for revealing the mechanisms underlying atropine-induced toxicity and may also provide various clues for further research.
\end{abstract}

\section{Introduction}

Atropine sulphate, a competitive antagonist for the muscarinic acetylcholine (ACh) receptors, was isolated from Atropa belladonna. It has been suggested that high potencies of atropine sulphate may have an influence on ACh-induced contraction of smooth muscles (1). Atropine is a competitive antagonist of the action of $\mathrm{ACh}$ and other muscarinic agonists, and it is shown to extract $\mathrm{Na}(+), \mathrm{K}(+), \mathrm{Mg}(2+)$ and $\mathrm{Ca}(2+)$ ions from an aqueous phase into an organic one with a preference for $\mathrm{Ca}(2+)$ ions (2).

Correspondence to: Dr Quan-Yong Huang, Department of Pathology, School of Basic Medical Sciences, Dali University, Xiaguan Wanhua Road, Dali, Yunnan 671000, P.R. China

E-mail: hqy0726@126.com

Key words: E-cadherin, 5-hydroxytryptamine, atropine-induced toxicity
Atropine exhibits diverse effects on the cardiovascular system. It prevents $\alpha 2$-agonist-induced bradycardia and increases the severity and duration of hypertension (3). Atropine was found to reduce discharge rates and reverse the increase in spontaneous activity induced by ACh (4). Patients who received an overdose of atropine had worse signs and symptoms of cardiomyopathy (5). It has also been suggested that chronic atropine administration affects vasoactive intestinal polypeptide synthesis in rat heart atria and consequently modifies regulation of the heart rate (6). Additionally, routine prophylaxis with an anticholinergic agent was found to aid in the prevention of sinus bradycardia during urological laparoscopic surgery (7). Furthermore, it has been demonstrated that early i.v. administration of high-dose atropine can prevent neurogenic pulmonary edema development on the basis of the prevention of baroreflex-induced bradycardia (8).

Atropine, a non-selective muscarinic receptor antagonist, is currently the most potent agent used to prevent myopia in animal models and children (9). It has been reported that regular topical administration of $0.025 \%$ atropine eye drops can prevent myopia onset and myopic shift in premyopic school children for a 1-year period (10).

However, numerous side effects attributed to atropine are commonly reported. Atropine has the potential to cause central toxicity which may complicate the management of cardiomyopathy (11). It has been reported that atropine may cause aspiration pneumonia through induction of the swallowing disorder and inhibition of the cough reflex (12), and it is associated with a higher incidence of a transient rash (13).

It has been shown that atropine-induced toxicity is involved in alterations in enzyme activities and nitric oxide (14); however, little is known concerning the changes in E-cadherin and 5-hydroxytryptamine (5-HT) expression in the heart having undergone atropine-induced toxicity.

The present study used immunohistochemistry to evaluate the expression of E-cadherin and 5-HT in the heart, and determine whether protein expression is altered upon atropine-induced toxicity.

\section{Materials and methods}

Sixteen healthy adult male Wistar rats, weighing 210-250 g, were used in this study. All animals were accommodated in 
cages in an environmentally controlled room at an ambient temperature of $20-26^{\circ} \mathrm{C}$ and relative humidity of $30-70 \%$, respectively, under a 12-h light/dark cycle (lights on from 6:00-18:00 h). Rats were fed with a standard pellet chow and water throughout the experimental period. All procedures described in this study were approved by the Ethics Committee of Sun Yat-Sen University.

Animals and study design. Animals were divided randomly into two groups, each with 8 rats. Atropine group animals were treated by an intraperitoneal (i.p.) injection of atropine at a dose of $15 \mathrm{mg} / \mathrm{kg} /$ day for 7 days; the control group animals were treated with normal saline for 7 days in equal volumes as for the experimental groups (15).

After 7 days of treatment with atropine $(15 \mathrm{mg} / \mathrm{kg} / \mathrm{day})$, the control and experimental animals were anesthetized with xylazine $(10 \mathrm{mg} / \mathrm{kg})$ and ketamine $\mathrm{HCl}(50 \mathrm{mg} / \mathrm{kg})$. Anesthetized rats were sacrificed and a thoracotomy was performed. The heart was harvested, fixed in phosphate-buffered $10 \%$ formalin, embedded in paraffin wax, and sectioned $(4 \mu \mathrm{m})$ for light microscopy and immunohistochemical examination.

Histopathologic examinations. Heart specimens from each group were removed to be examined histopathologically. After the heart tissues were fixed in $10 \%$ formalin and embedded in parafin, tissue sections were coronally sectioned to a thickness of $4 \mu \mathrm{m}$, and stained with hematoxylin and eosin. The slides were coded, and semi-quantitative analysis of the sections was performed without knowledge of the treatment protocol. In these tissues, pathological changes were evaluated $(16,17)$.

Tissue sections and immunohistochemical staining. All rat hearts were immersed in $4 \%$ formaldehyde buffered with phosphate-buffered saline (PBS, $\mathrm{pH} 7.2)$, and then embedded in paraffin. Tissue sections $(4 \mu \mathrm{m})$ were prepared for immunohistochemical staining. After deparaffinization, all sections were immersed in $0.3 \% \mathrm{H}_{2} \mathrm{O}_{2}$-PBS for $10 \mathrm{~min}$ and incubated with PBS containing $1 \%$ normal goat serum to reduce non-specific reactions. After incubation with either rabbit anti-E-cadherin or anti-5-HT (Santa Cruz Biotechnology, USA) overnight at $4^{\circ} \mathrm{C}$, tissue sections were washed three times in PBS and incubated with biotin-conjugated secondary antibody for $1 \mathrm{~h}$ at room temperature according to the manufacturer's instructions. Then the specimens were incubated with peroxidase-conjugated avidin-biotin for $30 \mathrm{~min}$ at room temperature. After washing, the sections were incubated with diaminobenzidine (DAB) as chromogen, and counterstained with hematoxylin (18-20). Negative control sections were performed using PBS buffer instead of the primary antibody.

Total integrated optical density (IOD), a parameter representing the expression levels of E-cadherin and 5-HT in cardiac tissue, was determined using a cast-grid microscope (MetaMorph/DP10/Bx41, UIC/Olympus, US/JP) together with an image-analysis program (MetaMorph offline, version 4.65). Under magnification of $\mathrm{x} 400$, five images were captured in each immunostained section, and the average was calculated $(19,20)$.

Statistical analyses. Statistical analyses were performed using SPSS 11.0 software. The results were presented as the
Table I. Integrated optical density (IOD) of E-cadherin and 5 -HT in the heart of rats.

\begin{tabular}{lcc}
\hline Groups & E-cadherin & $5-\mathrm{HT}$ \\
\hline Control group & $0.0136 \pm 0.00057$ & $0.0145 \pm 0.00026$ \\
Atropine group & $0.0069 \pm 0.00028$ & $0.0056 \pm 0.00068$
\end{tabular}

The integrated optical density (IOD) per field is proportional to the total amount of staining. Total E-cadherin IOD expression in rats having undergone atropine intoxication was significantly lower compared with the control hearts $(\mathrm{P}<0.05)$. Total 5-HT IOD expression in rat hearts subjected to atropine intoxication was significantly lower when compared to the control $(\mathrm{P}<0.05)$.

mean \pm SEM. The sources of variation were analyzed by unpaired Student's t-test. A P-value of $<0.05$ was considered to denote statistical significance.

\section{Results}

Histological examination. Routine histological examination revealed slight morphologic change in the rat hearts from each group (data not shown).

Immunohistochemical staining. Immunohistochemical staining was performed on formalin-fixed, paraffin-embedded sections from all parts of the heart including the conduction system.

Expression of E-cadherin protein. Positive staining for the E-cadherin antibody was detected mainly in the extracellular matrix. The E-cadherin antibody was manifested as fine brown granularity. Two observers examined the sections independently.

The photomicrographs in Fig. 1 display the positive expression of E-cadherin in the control (Fig. 1A) and atropine-injured (Fig. 1B) rat hearts. Total IOD expression of E-cadherin in the rat hearts from each group is documented in Table I. Total IOD expression of E-cadherin in the hearts from rats having undergone atropine intoxication was significantly lower than that in the control $(\mathrm{P}<0.05)$.

Expression of 5-HT protein. The 5-HT staining was detected mainly in the extracellular matrix, and positive staining for 5-HT was manifested as fine brown granularity.

The distribution of 5-HT in the rat hearts of the control and atropine groups is shown in Fig. 2A and B, respectively. Total 5-HT IOD in the heart of animals subjected to atropine intoxication was significantly lower than that of the control hearts (Table I) $(\mathrm{P}<0.05)$.

\section{Discussion}

Although atropine is widely used for fiberoptic bronchoscopy and helps by drying secretions, producing bronchodilatation, or preventing vasovagal reactions, the undesirable associated side effects may markedly decrease the quality of life of patients. 

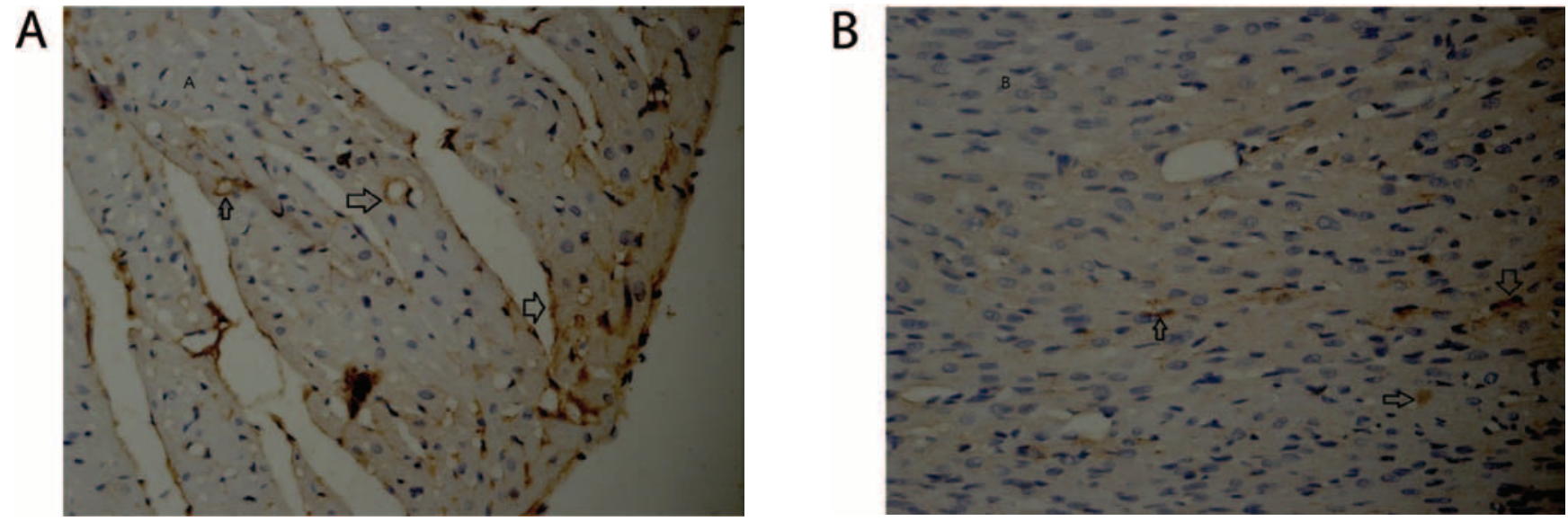

Figure 1. Effect of atropine exposure on E-cadherin expression in rat hearts. Photomicrographs display the representative distribution of E-cadherin-positive expression in rat hearts from the (A) control and (B) atropine-exposed group. All photomicrographs were captured at a magnification of $\mathrm{x} 400$. Positive immunostaining appears as brown staining (arrow).
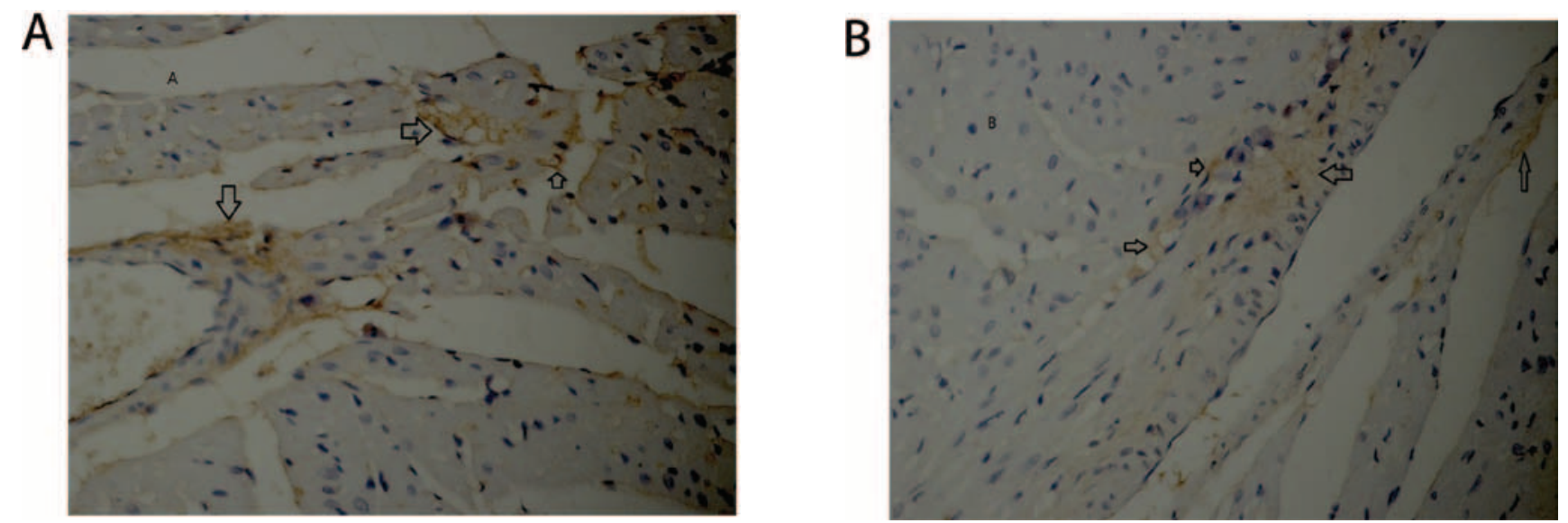

Figure 2. Effect of atropine exposure on 5-HT expression in rat hearts. Photomicrographs reveal a representative distribution of positive 5-HT expression in rat hearts from the (A) control and (B) atropin-exposed group. All photomicrographs were taken at a magnification of x400. Positive immunostaining appears as brown staining (arrow).

It has been suggested that dysregulation of E-cadherin is involved in carcinoma development (21). E-cadherin is a cell-cell adhesion protein and tumor suppressor that is silenced in many malignancies, and it is thought to suppress tumor cell growth by antagonizing $\beta$-catenin signaling (22). Alterations in E-cadherin are associated with certain types of cancer. Regulated adhesion between cells and their environment is critical for normal cell migration, and it has been suggested that mediated reduction in $\mathrm{H}_{2} \mathrm{O}_{2}$ is required to maintain E-cadherin protein levels in the early embryo (23).

E-cadherin has been proven to be widely downregulated and tightly associated with tumor invasion and metastasis in multiple human cancer types (24). Previous research has indicated that loss of E-cadherin induces possible pre-cancerous lesions in the gastric mucosa but may not be sufficient for its malignant conversion (25). However, research has shown that downregulation of E-cadherin plays important roles in tumor progression and metastasis. It has been shown that loss of E-cadherin contributes to both mammary tumor initiation and metastasis (26), and the expression of E-cadherin correlates with the progression and metastasis of gastric cancer (27).
Meanwhile, E-cadherin is a tumor-suppressor gene involved in epithelial cell-cell interactions and plays important roles in the etiology of gastric cancer (28). Similarly, it has been reported that reduced levels of E-cadherin in nasopharyngeal carinoma may play an important role in invasion and metastasis (29). Decreased E-cadherin levels in the heart noted in our results suggest that the tissues were extensively damaged after undergoing atropine-induced toxicity. The present study indicates that atropine-induced toxicity causes decreased E-cadherin expression that is related to impaired heart function.

The biogenic amine serotonin 5-HT plays a key role in the regulation and modulation of various physiological and behavioral processes (30). It has been recognized that 5-HT plays important roles in peripheral tissues and vascular cells and is involved in coagulation, hypertension, inflammation, healing and protection (31). It also has been shown that descending serotonergic 5-HT system is shown to be plastically altered under pathological conditions, for example, the enhancement of 5-HT-induced contraction in mesenteric arteries (32). Furthermore, it has been suggested that 5 -HT is involved in the fine adjustment at several brain centers including the 
core of the mammalian circadian timing system (33). It has been reported that 5-HT is a major factors in the pathogenesis of pulmonary arterial hypertension (34). In addition, the endogenous brain serotonin 5-HT system is believed to have an important influence in mediating drug reward and seeking mechanisms (35). Furthermore, previous research has shown that a variation in expression of the 5-HT transporter (5-HTT) is linked to the risk of psychiatric illness (36).

It has been suggested that stress plays a key role in the modulation of 5-HT. For example, stress is known to activate the central 5-HT system, and this is probably part of a coping response involving several 5-HT receptors (37). The serotonin 5-HT is capable of reducing the oxidative burst of phagocytes (38). Melatonin may act as a modulator of excess 5-HT release from colonic mucosa (39). Moreover, decreased 5-HT in the heart noted in our study suggests that the tissues were extensively damaged after undergoing atropine-induced toxicity. The present study indicates that atropine-induced toxicity causes decreased 5-HT expression that is related to impaired heart function.

In the present study, our results demonstrated that cardiac protein expression of E-cadherin and 5-HT is altered after atropine-induced toxicity in the rat. After undergoing atropineinduced toxicity, protein expression of E-cadherin and 5-HT was significantly reduced. The present study indicates that atropine-induced toxicity causes different alterations in heart proteins that may be related to cardiac-function deterioration.

In conclusion, the present findings indicate that such alterations would be reflected in abnormal cardiac function, and the studied proteins may be useful for revealing the mechanisms underlying atropine-induced toxicity and may also provide certain clues for further research.

\section{Acknowledgements}

This study was supported by a grant from the Natural Science Foundation of Guangdong Province, China (no. 4203003) and Guangdong Medical College (no. XQ0426).

\section{References}

1. Siegling-Vlitakis C, Martens H and Ludtke R: In vitro examination of potentized atropine sulfate dilutions on the contractility of the isolated rat ileum. J Altern Complement Med 15: 1121-1126, 2009.

2. Rabi L, Moutaouakkil A and Blaghen M: Ionophoric properties of atropine: complexation and transport of $\mathrm{Na}^{+}, \mathrm{K}^{+}, \mathrm{Mg}^{2+}$ and $\mathrm{Ca}^{2+}$ ions across a liquid membrane. Nat Prod Res 22: 547-553, 2008.

3. Alvaides RK, Neto FJ, Aguiar AJ, Campagnol D and Steagall PV: Sedative and cardiorespiratory effects of acepromazine or atropine given before dexmedetomidine in dogs. Vet Rec 162: 852-856, 2008

4. Drexler B, Antkowiak B, Thiermann H and Grasshoff C: Atropine increases sevoflurane potency in cortical but not spinal networks during cholinergic overstimulation. Toxicology 268: 98-103, 2010

5. Sandhu G, Servetnyk Z, Croitor S and Herzog E: Atropine aggravates signs and symptoms of Takotsubo cardiomyopathy. Am J Emerg Med 28: 255-258, 2010.

6. Kuncova J, Faitova S, Capouch J, Stengl M and Slavikova J: Chronic atropine administration diminishes the contribution of vasoactive intestinal polypeptide to heart rate regulation. Physiol Res 57: 827-837, 2008.

7. Aghamohammadi H, Mehrabi S and Mohammad Ali Beigi F: Prevention of bradycardia by atropine sulfate during urological laparoscopic surgery: a randomized controlled trial. Urol J 6: 92-95, 2009
8. Sedy J, Zicha J, Kunes J and Sykova E: Atropine may prevent the development of neurogenic pulmonary edema. Med Hypotheses 73: 42-44, 2009

9. Diether S, Schaeffel F, Lambrou GN, Fritsch C and Trendelenburg AU: Effects of intravitreally and intraperitoneally injected atropine on two types of experimental myopia in chicken. Exp Eye Res 84: 266-274, 2007.

10. Fang PC, Chung MY, Yu HJ and Wu PC: Prevention of myopia onset with $0.025 \%$ atropine in premyopic children. J Ocul Pharmacol Ther 26: 341-345, 2010.

11. Arendse R and Irusen E: An atropine and glycopyrrolate combination reduces mortality in organophosphate poisoning. Hum Exp Toxicol 28: 715-720, 2009.

12. Tsubouchi T, Tsujimoto S, Sugimoto S, Katsura Y, Mino T and Seki T: Swallowing disorder and inhibition of cough reflex induced by atropine sulfate in conscious dogs. J Pharmacol Sci 106: 452-459, 2008.

13. Heinz P, Geelhoed GC, Wee C and Pascoe EM: Is atropine needed with ketamine sedation? A prospective, randomised, double blind study. Emerg Med J 23: 206-209, 2006.

14. Kose A, Gunay N, Yildirim C, Tarakcioglu M, Sari I and Demiryurek AT: Cardiac damage in acute organophosphate poisoning in rats: effects of atropine and pralidoxime. Am J Emerg Med 27: 169-175, 2009.

15. Pazoki-Toroudi HR, Ajami M and Habibey R: Pre-medication and renal pre-conditioning: a role for alprazolam, atropine, morphine and promethazine. Fundam Clin Pharmacol 24: 189-198, 2010.

16. Helin HO, Lundin ME, Laakso M, Lundin J, Helin HJ and Isola J: Virtual microscopy in prostate histopathology: simultaneous viewing of biopsies stained sequentially with hematoxylin and eosin, and alpha-methylacyl-coenzyme A racemase/p63 immunohistochemistry. J Urol 175: 495-499, 2006.

17. De Rossi A, Rocha LB and Rossi MA: Application of fluorescence microscopy on hematoxylin and eosin-stained sections of healthy and diseased teeth and supporting structures. J Oral Pathol Med 36: 377-381, 2007.

18. Smith PS, Parkinson IH and Leong AS: Principles of ploidy analysis by static cytometry. Clin Mol Pathol 49: M104-M111, 1996.

19. Dong J, Yin H, Liu W, Wang P, Jiang Y and Chen J: Congenital iodine deficiency and hypothyroidism impair LTP and decrease $\mathrm{C}$-fos and C-jun expression in rat hippocampus. Neurotoxicology 26: 417-426, 2005.

20. van Kuijk AW, Gerlag DM, Vos K, et al: A prospective, randomised, placebo-controlled study to identify biomarkers associated with active treatment in psoriatic arthritis: effects of adalimumab treatment on synovial tissue. Ann Rheum Dis 68: 1303-1309, 2009.

21. Pap Z, Pavai Z, Denes L, Kovalszky I and Jung J: An immunohistochemical study of colon adenomas and carcinomas: E-cadherin, Syndecan-1, Ets-1. Pathol Oncol Res 15: 579-587, 2009.

22. Lau MT, Klausen C and Leung PC: E-cadherin inhibits tumor cell growth by suppressing PI3K/Akt signaling via $\beta$-cateninEgrl-mediated PTEN expression. Oncogene 30: 2753-2766, 2011.

23. DeGennaro M, Hurd TR, Siekhaus DE, Biteau B, Jasper H and Lehmann R: Peroxiredoxin stabilization of DE-cadherin promotes primordial germ cell adhesion. Dev Cell 20: 233-243, 2011.

24. Ran Y, Wu S and You Y: Demethylation of E-cadherin gene in nasopharyngeal carcinoma could serve as a potential therapeutic strategy. J Biochem 149: 49-54, 2011.

25. Mimata A, Fukamachi H,Eishi Y and Yuasa Y: Loss of E-cadherin in mouse gastric epithelial cells induces signet ring-like cells, possible precursor lesion of diffuse gastric cancer. Cancer Sci 102: 942-950, 2011.

26. Derksen PW, Braumuller TM, van der Burg E, et al: Mammaryspecific inactivation of E-cadherin and p53 impairs functional gland development and leads to pleomorphic invasive lobular carcinoma in mice. Dis Model Mech 4: 347-358, 2011.

27. Uchikado Y, Okumura H, Ishigami S, et al: Increased Slug and decreased E-cadherin expression is related to poor prognosis in patients with gastric cancer. Gastric Cancer 14: 41-49, 2011.

28. Wang Q, Gu D, Wang M, Zhang Z, Tang J and Chen J: The E-cadherin (CDH1) -160C $>$ A polymorphism associated with gastric cancer among Asians but not Europeans. DNA Cell Biol 30: 395-400, 2011.

29. Galera-Ruiz H, Rios MJ, Gonzalez-Campora R, et al: The cadherin-catenin complex in nasopharyngeal carcinoma. Eur Arch Otorhinolaryngol 268: 1335-1341, 2011. 
30. Blenau W and Thamm M: Distribution of serotonin (5-HT) and its receptors in the insect brain with focus on the mushroom bodies: lessons from Drosophila melanogaster and Apis mellifera. Arthropod Struct Dev 40: 381-394, 2011.

31. Mercado CP, Ziu E and Kilic F: Communication between 5-HT and small GTPases. Curr Opin Pharmacol 11: 23-28, 2011.

32. Matsumoto T, Kobayashi T, Ishida K, Taguchi K and Kamata K: Enhancement of mesenteric artery contraction to 5-HT depends on Rho kinase and Src kinase pathways in the ob/ob mouse model of type 2 diabetes. Br J Pharmacol 160: 1092-1104, 2010.

33. Cavalcante JS, de Pontes AL, Engelberth RC, et al: 5-HT(1B) receptor in the suprachiasmatic nucleus of the common marmoset (Callithrix jacchus). Neurosci Lett 488: 6-10, 2011.

34. Cogolludo A and Perez-Vizcaino F: 5-HT receptors and K(V) channel internalization. Adv Exp Med Biol 661: 391-401, 2010.

35. Fijal K, Pachuta A, McCreary AC, et al: Effects of serotonin $(5-\mathrm{HT})(6)$ receptor ligands on responding for cocaine reward and seeking in rats. Pharmacol Rep 62: 1005-1014, 2010.
36. Jennings KA, Lesch KP, Sharp T and Cragg SJ: Non-linear relationship between 5-HT transporter gene expression and frequency sensitivity of 5-HT signals. J Neurochem 115: 965-973, 2010.

37. Mongeau R, Martin CB, Chevarin C, et al: 5 -HT2C receptor activation prevents stress-induced enhancement of brain 5-HT turnover and extracellular levels in the mouse brain: modulation by chronic paroxetine treatment. J Neurochem 115: 438-449, 2010.

38. Pracharova L, Okenkova K, Lojek A and Ciz M: Serotonin and its 5-HT(2) receptor agonist DOI hydrochloride inhibit the oxidative burst in total leukocytes but not in isolated neutrophils. Life Sci 86: 518-523, 2010.

39. Kojima S, Tohei A and Ikeda M: Melatonin inhibits tachykinin $\mathrm{NK}_{2}$ receptor-triggered 5-HT release from guinea pig isolated colonic mucosa. Br J Pharmacol 162: 1179-1185, 2011. 